\title{
An anticipative scheduling approach with controllable processing times
}

\author{
Sinan Gürel ${ }^{\mathrm{b}}$, Ersin Körpeoğlu ${ }^{\mathrm{a}}$, M. Selim Aktürk ${ }^{\mathrm{a}, *}$ \\ a Department of Industrial Engineering, Bilkent University, 06800 Bilkent, Ankara, Turkey \\ ${ }^{\mathrm{b}}$ University of Warwick, Centre for Discrete Mathematics and its Applications (DIMAP), Coventry CV4 7AL, UK
}

\section{A R T I C L E I N F O}

\section{Available online 16 September 2009}

Keywords:

Anticipative scheduling

Controllable processing times

Reactive scheduling

Match-up time

\begin{abstract}
A B S T R A C T
In practice, machine schedules are usually subject to disruptions which have to be repaired by reactive scheduling decisions. The most popular predictive approach in project management and machine scheduling literature is to leave idle times (time buffers) in schedules in coping with disruptions, i.e. the resources will be under-utilized. Therefore, preparing initial schedules by considering possible disruption times along with rescheduling objectives is critical for the performance of rescheduling decisions. In this paper, we show that if the processing times are controllable then an anticipative approach can be used to form an initial schedule so that the limited capacity of the production resources are utilized more effectively. To illustrate the anticipative scheduling idea, we consider a non-identical parallel machining environment, where processing times can be controlled at a certain compression cost. When there is a disruption during the execution of the initial schedule, a match-up time strategy is utilized such that a repaired schedule has to catch-up initial schedule at some point in future. This requires changing machine-job assignments and processing times for the rest of the schedule which implies increased manufacturing costs. We show that making anticipative job sequencing decisions, based on failure and repair time distributions and flexibility of jobs, one can repair schedules by incurring less manufacturing cost. Our computational results show that the match-up time strategy is very sensitive to initial schedule and the proposed anticipative scheduling algorithm can be very helpful to reduce rescheduling costs.
\end{abstract}

(c) 2009 Elsevier Ltd. All rights reserved.

\section{Introduction}

Unexpected events such as machine breakdowns or new job arrivals necessitate rescheduling remaining jobs in a schedule. Processing time controllability provides us flexibility in rescheduling against unexpected disruptions by allowing changes on the processing times of the jobs. However, the performance of rescheduling decisions, such as replanning the processing times or reallocating jobs between machines, highly depends on the state of the schedule at the time of disruption. Thus, it is critical to prepare initial schedules by considering possible disruption times and the ability of jobs to absorb disruptions. In this study, we develop an anticipative scheduling approach to form an initial schedule that could improve the performance of rescheduling decisions with controllable processing times.

To illustrate the anticipative scheduling idea, we design a scheduling algorithm for a set of jobs to be scheduled on parallel non-identical machines with given machining time capacities on each machine. Initial objective for this problem is to minimize the

\footnotetext{
* Corresponding author. Fax: +90312266 4054 .

E-mail addresses: Sinan.Gurel@wbs.ac.uk (S. Gürel), ersink@bilkent.edu.tr (E. Körpeoglu), akturk@bilkent.edu.tr (M.S. Aktürk).
}

total manufacturing cost of the jobs. We first find the optimal machine-job assignment and the optimal compression levels on the processing times of the jobs. Having found the machine-job assignments, the problem is to find a job sequence on each machine. We consider the situation that if a machine breakdown occurs on one of the machines, a reactive scheduling problem is solved and the remaining schedule is repaired. We assume that failure and repair times are uncertain with given probability distributions. In the considered reactive scheduling problem, the objective is to minimize the manufacturing cost increase due to disruption, denoted as rescheduling cost, subject to the condition that the repaired schedule has to match up with the initial schedule at a given time point after disruption. We provide a scheduling algorithm which determines a job sequence on each machine by considering possible downtime periods on the machines along with rescheduling cost minimization objective.

\subsection{Literature}

In the scheduling literature, reactive and predictive scheduling approaches have been considered extensively. In those studies usually the aim is to prepare an initial schedule in such a way that the schedule can be repaired with simple 
adjustments and within a slight performance degradation. Aytug et al. [1] gave an extensive literature survey on scheduling under uncertainty and generating robust schedules. Jensen [2] defined a robust schedule as the one which performs good when there was a disruption and the schedule was right shifted. Leon et al. [3] considered finding robust schedules in a job shop scheduling environment which is subject to a single disruption. They proposed a genetic algorithm to minimize expected makespan and expected delay measures. To the best of our knowledge, studies in the literature assume fixed processing times. Here, we consider anticipative scheduling with controllable processing times.

\subsubsection{Idle time insertion}

In order to minimize the effects of possible disruptions on a schedule, a well known predictive approach is inserting idle times in it, so that disruptions can be absorbed without disturbing the system. Almost all of the existing reactive scheduling strategies (including match-up and right-shift scheduling techniques) try to accommodate disruptions by using the available idle times on initial schedules. Inserting idle times, as a predictive scheduling approach, is first proposed by Mehta and Uzsoy [4] for a job-shop scheduling problem. Recently Leus and Herroelen [5] presented a new model for single machine scheduling problems with stability objective and a common deadline, and proposed a branch and bound algorithm for an approximate formulation of the model to determine when and where to place an inserted idle time. Their algorithm gives the optimal job sequence and the optimal length of idle time following each job in the schedule when exactly one job deviates from its expected processing time. Yang and Geunes [6] considered inserting idle times on a single machine scheduling problem where there exists uncertain future jobs that may arrive. They proposed a heuristic dynamic programming algorithm to minimize the expected sum of tardiness cost, the disruption cost and the wasted idle time cost. A similar idea (e.g. inserting a time buffer to protect a deterministic baseline schedule in order to cope with uncertainties) is also proposed for the project management problems, called buffer sizing, in the critical chain scheduling and buffer management (CC/BM) software as discussed in Herroelen and Leus [7]. In rescheduling with fixed processing times, inserting idle time is an efficient predictive approach. However, in case of controllable processing times, when the machining time capacity is limited and fully utilized, inserting idle times into a schedule require applying extra compression on the processing times of jobs. This increases compression costs. If no disruption occurs or if a disruption occurs after the inserted idle times, then the inserted idle time becomes useless. If the processing times are controllable, an alternative rescheduling approach to inserting idle times is reacting to disruptions by replanning the processing times. Consequently, the limited capacity of production resources are utilized more effectively.

In any idle time insertion approach, a critical decision to be made is when and how much idle time should be placed into an initial schedule so that the new schedule achieved by rescheduling after a disruption has the best scheduling performance. Analogously, in rescheduling with controllable processing times, it is critical to find the positions of the jobs in the initial schedule in an appropriate order so that a possible disruption is absorbed immediately and with a reasonable manufacturing cost increase. Therefore, we propose a new anticipative scheduling algorithm to form an initial schedule that takes flexibility of jobs along with probability distributions of failure and repair time of machines into account. Proposed flexibility measures estimate the ability of the jobs to absorb disruptions with less compression and reallocation costs, so that we schedule the most flexible jobs to the time zones where the downtime probability of a machine is higher.

\subsubsection{Controllable processing times}

A well known example to a controllable processing time is the processing time of CNC machining operations in flexible manufacturing systems. We can control the processing time of a job by setting the cutting speed and/or the feed rate on the machine. In turning operation as you increase the cutting speed and the feed rate, the processing time of the operation is compressed, whereas the compression cost of the operation is increased due to increased tooling costs [8]. Shabtay and Steiner [9] give an extensive literature review on the scheduling problems with controllable processing times. To the best of our knowledge, generating flexible schedules for the scheduling environments with controllable processing times has not been considered in the literature yet. Our work is the first attempt to employ anticipative scheduling with controllable processing times.

\subsubsection{Match-up scheduling}

When a disruption occurs, in order to stay consistent with the initial schedule, a critical rescheduling goal is to catch up with the initial schedule as soon as possible. The new schedule catches up with the initial schedule at the time point where the new schedule is exactly at the same state as the initial schedule. This time point is called the match-up time. Minimizing the match-up time helps to reduce the effects of a disruption on the production plan and on the schedules at the other stages of the production. For example, an extensive change in the completion times of jobs in the schedule of a department may cause unavailability of parts for the scheduled production in another department. In the literature, there exists few match-up scheduling studies such as Bean et al. [10] and Aktürk and Görgülü [11], which consider heuristic approaches to find match-up times under the existence of inserted idle times and fixed processing times. In rescheduling with controllable processing times, catching up an initial schedule earlier is possible by extensively compressing the jobs that are scheduled just after the disruption. With convex compression costs, absorbing a downtime by compressing a smaller set of jobs in the schedule results higher compression costs. Hence, there is a trade-off between the match-up time and the cost of the new schedule. Aktürk et al. [12] considered match-up time minimization and cost minimization problems for a parallel machine environment and showed the trade-off between two objectives.

\subsection{Contribution}

In this study, we introduce an anticipative scheduling approach with controllable processing times. We show that using the reactive scheduling objective and constraints, uncertainty data for downtimes, manufacturing cost and processing time controllability simultaneously, one can prepare initial schedules which could result improved rescheduling cost performance in case of a disruption.

As a specific problem we consider generating flexible initial schedules for the manufacturing cost objective by using an anticipative scheduling approach. For the rescheduling problem, we will consider minimizing rescheduling cost subject to a given match-up time point. We show that the rescheduling cost objective is quite sensitive to the set of jobs that are affected by the machine breakdown. Our scheduling algorithm uses the failure and repair time distributions and the manufacturing cost functions of the jobs in order to find the initial schedules which can be repaired at lower rescheduling cost levels. The proposed approach in this study incurs no additional cost in terms of 
match-up time and manufacturing cost, but gives less rescheduling costs in case of a machine breakdown. Our computational experiments show that our approach can achieve an average improvement of $31 \%$ in rescheduling costs.

\subsection{Organization}

In Section 2, we define the considered scheduling environment, formulate the rescheduling cost minimization problem and then discuss the related scheduling problem. In Section 3, we introduce our anticipative scheduling algorithm. We first introduce machine-job allocation problem briefly, then present a probabilistic analysis and discuss proposed flexibility measures. Finally, we give a probabilistic sequencing algorithm for the cost minimization problem. Section 4 gives the results of the computational experiments and we conclude with Section 5.

\section{Rescheduling cost minimization problem}

We consider $n$ jobs to be processed on $m$ non-identical parallel CNC machines. Processing time of job $j$ on machine $i$ is $p_{i j}^{u}$. Processing time of a job on machine can be compressed. Amount of compression $y_{i j}$ is a decision variable and has an upper bound $u_{i j}$. Manufacturing cost of job $j$ on machine $i$ is $c_{i j}$. Compression cost function for job $j$ on machine $i$ is $f_{i j}\left(y_{i j}\right)$. On each machine, there is a given available machining time capacity $D_{i}$. For the considered rescheduling problem, an initial machine-job assignment, denoted by $\mathcal{A}$, is obtained by solving a minimum cost machine-job assignment problem which will be introduced in Section 3.1.

Given $\mathcal{A}$, an initial schedule, called $\mathcal{S}$, with the start and end times of jobs on each machine is to be formed by finding a job sequence on each machine. Different disruptions may occur to a schedule $\mathcal{S}$ during its execution. In this study, we assume that a breakdown could occur on one of the machines at an uncertain time. We also assume that since the failed machine has to be fixed, it will be down for an uncertain amount of time which will be known at the time of breakdown. If the breakdown occurs in the middle of the processing a job, the job has to be reprocessed in its entirety. This situation is called the preempt-repeat case in the literature.

Given such a downtime period on one of the machines, $\mathcal{S}$ is no longer executable. A subset of jobs in $\mathcal{S}$ has already been finished before the disruption. We assume that the jobs being processed on the machines other than the disrupted machine at the time of breakdown will finish their process as planned in $\mathcal{S}$. The other jobs which have not started processing yet at the time of breakdown and the job which is disrupted by the breakdown on the failed machine have to be rescheduled. These jobs are either to be reallocated to other machines and/or replanned to calculate their new processing times.

We consider a rescheduling cost minimization problem which is to be solved after a breakdown occurs. As one of the machines is disrupted and the schedule for the remaining jobs has to be repaired, one can look for alternative machine-job assignments and processing time decisions. Repaired schedule is required to satisfy the scheduling and processing time related constraints at a minimum rescheduling cost. It is also required that the repaired schedule catches up the initial schedule as soon as possible after a breakdown. Therefore, this problem could be formulated as to minimize the rescheduling cost of remaining jobs for a given limit on the match-up time. In this problem, a match-up time on a machine implies that the schedule, i.e. the job sequence and startend times of the jobs, following the match-up point is exactly the same as in initial schedule $\mathcal{S}$. As we consider a non-preemptive rescheduling environment, we select match-up times out of the start times of the jobs previously determined in $\mathcal{S}$.

\subsection{Manufacturing cost function}

The manufacturing cost of a job on a machine is a fixed amount $c_{i j}$, which is the cost if the job is operated at $p_{i j}^{u}$, plus the compression cost which is incurred if the processing time of the job is compressed. Compressing the processing time of a job requires using additional resource. As we increase the cutting speed and/or feed rate on a CNC machine, the tool life is reduced and hence the manufacturing cost is increased. The compression cost of each job can be expressed as a function of $y \geq 0$ as

$f(y)=k y^{(a / b)}$

where $a \geq b>0$ and $k>0$ so that $f$ is increasing and convex. As we decrease the processing time of a job, it requires more additional resource to compress it further. As discussed in Kayan and Aktürk [13], in turning operation, the length and the diameter of the job, the required surface roughness, machine horsepower, and the required tool type determine the cost coefficients $k, a$, and $b$ for each machine-job pair.

\subsection{Rescheduling problem formulation}

In the rescheduling cost minimization problem, for each job to be rescheduled, a machine-job assignment decision has to be made. $x_{i j}$ is the assignment variable which is 1 if job $j$ is assigned to machine $i$ and 0 otherwise. Also, for each job a new compression amount $\left(y_{i j}\right)$ has to be determined. Given an upper bound $W$ on the match-up times, one can set the match-up time on machine $i$ to be $W_{i}=\max _{j \in J_{i}}\left\{s_{j}: s_{j} \leq W\right\}$. This is because the match-up times can be selected out of the start times of the jobs in the initial schedule. We define the set of jobs to be rescheduled as $J^{W}$, i.e. set of jobs that precede selected match-up times on the machines. Furthermore, we define $C_{j}^{\mathcal{S}}$ to be the manufacturing cost of job $j$ in $\mathcal{S}$. We denote the machining time capacity on machine $i$ used by $\mathcal{S}$ as $D_{i}^{\mathcal{S}}$. Then, we can formulate the problem of minimizing total manufacturing cost of jobs in $J^{W}$ with given match-up times as

$$
\begin{aligned}
& \min \sum_{i} \sum_{j \in J^{W}}\left(c_{i j} x_{i j}+f_{i j}\left(y_{i j}\right)\right)-\sum_{j \in J^{W}} C_{j}^{\mathcal{S}} \\
& \text { s.t. } \sum_{j \in J^{W}}\left(p_{i j}^{u} x_{i j}-y_{i j}\right) \leq W_{i}-D_{i}^{\mathcal{S}}, \quad i=1, \ldots, m, \\
& (\mathrm{RCM}) \quad y_{i j} \leq x_{i j} u_{i j}, \quad i=1, \ldots, m \text { and } j \in J^{W}, \\
& \sum_{i=1}^{m} x_{i j}=1, \quad j \in J^{W}, \\
& \quad x_{i j} \in\{0,1\}, \quad y_{i j} \in \mathbb{R}_{+}, \quad i=1, \ldots, m \text { and } j \in J^{W} .
\end{aligned}
$$

In this formulation, constraint set (1) guarantees that completion times of jobs in $J^{W}$ do not exceed the match-up time in the new schedule. Constraint set (2) is the variable upper bounding constraints on the amount of compression, guaranteeing that processing time of a job on a machine can be compressed only if the job is assigned on that machine and also the compression cannot be greater than the upper bound $u_{i j}$. Constraint set (3) assigns each job in set $J^{W}$ to a machine. RCM is a mixed integer nonlinear programming problem for which Aktürk et al. [14] provided a strengthened conic quadratic formulation. Therefore, it can be solved efficiently by a commercial branch-and-bound 
software which employs second-order cone programming algorithms in solving subproblems.

Given the rescheduling problem above we focus on developing an anticipative scheduling approach to form an initial schedule. Given $\mathcal{A}$, under the assumption of a single disruption on one of the machines and the assumption that RCM problems to be solved to reschedule the remaining jobs, the problem that we deal with is to make the job sequencing decisions on each machine to form the initial schedule $\mathcal{S}$ so that the optimal solution of RCM can be improved. In the next section, we explore the probabilistic nature of downtime period on a machine and propose flexibility measures which estimate the ability of the jobs to absorb downtimes.

\section{Anticipative scheduling algorithm}

We develop an anticipative scheduling approach to form an initial schedule. It is uncertain which machine will fail, at what time and how long it will take to repair a failed machine. We assume that the probability distributions for failure and repair times are known. When a disruption occurs it is critical to absorb the downtime as soon as possible and at minimum rescheduling cost. Therefore, it is critical which jobs are scheduled at and immediately after the downtime interval. So, we provide a set of flexibility measures to be evaluated for each job. We will use the flexibility measures in deciding which jobs are appropriate to schedule at risky time zones. An outline of proposed anticipative scheduling algorithm is given below.

Algorithm 1. Anticipative Scheduling Algorithm.

Step 1. Initial machine-job assignment, $\mathcal{A}$ : Find the minimum cost machine-job assignment for given jobs and machining time capacity levels $\left(D_{i}\right)$;

Step 2. Downtime probability: For each machine find the downtime probability function which gives the probability that the machine will be down at a time point $t$;

Step 3. Flexibility measures: Develop a flexibility measure $F_{j}$ for each job with respect to:

- Processing time,

- Compressibility range,

- Second derivative of the compression cost function,

- Average slope of the compression cost function,

- Machine-job reallocation cost estimate;

Step 4. Probabilistic sequencing algorithm: Sequence the jobs on the machines by placing the most flexible job, i.e. job with the highest $F_{j}$, to the time zone where the machine is most likely to be down.

\subsection{Initial machine-job assignment}

As a first step of our anticipative scheduling algorithm, we solve a machine-job assignment problem to minimize the total manufacturing cost of given $n$ jobs to be completed on $m$ nonidentical machines. A mathematical formulation of the problem is as follows:

$$
\begin{array}{ll}
\min & \sum_{i=1}^{m} \sum_{j=1}^{n}\left(c_{i j} x_{i j}+f_{i j}\left(y_{i j}\right)\right) \\
\text { s.t. } & \sum_{j=1}^{n}\left(p_{i j}^{u} x_{i j}-y_{i j}\right) \leq D_{i}, \quad i=1, \ldots, m,
\end{array}
$$

$$
\begin{aligned}
& \text { (MJA) } \quad y_{i j} \leq x_{i j} u_{i j}, \quad i=1, \ldots, m, j=1, \ldots, n, \\
& \sum_{i=1}^{m} x_{i j}=1, \quad j=1, \ldots, n, \\
& x_{i j} \in\{0,1\}, \quad y_{i j} \in \mathbb{R}_{+}, \quad i=1, \ldots, m, j=1, \ldots, n .
\end{aligned}
$$

The difference between MJA and RCM is that MJA is solved for $n$ jobs at the beginning when capacity on each machine is the initially available machining time $D_{i}$. MJA is a mixed-integer nonlinear programming problem which can be solved similar to the RCM problem by using the conic quadratic reformulation approach proposed by Aktürk et al. [14]. In this approach the first step is to move each convex function $f(y)$ in the objective into the constraint set. This is done by introducing auxiliary variables $(t \geq 0)$ to the problem. Then, objective function of the resulting formulation is

$\sum_{i=1}^{m} \sum_{j=1}^{n}\left(c_{i j} x_{i j}+t_{i j}\right)$.

For each $i, j$ pair, new formulation includes constraint

$y^{a} \leq t^{b}$.

Having variable upper bounding constraints (6) in the formulation, one can strengthen this inequality as follows:

$y^{a} \leq t^{b} x^{(a-b)}$,

which can be represented by second-order conic constraints. Computational study by Aktürk et al. [14] has shown that conic representation of strengthened formulation can be solved quite efficiently compared to conic representation of the original formulation. In this study, we have used this reformulation approach in solving MJA and RCM problems. Next, we define a downtime probability function and show how it is derived.

\subsection{Downtime probability}

Given the failure time and repair time distributions for a machine, one can calculate the probability that it will be down at a certain time $t$. Let $\mathcal{X}_{i}$ be the random variable defining the failure time of machine $i$ and $\mathcal{Y}_{i}$ be the random variable defining the repair time after a failure occurs, then we can define the probability that machine $i$ will be down at time $t$ as below:

$P_{i}^{d}(t)=P\left(\mathcal{X}_{i} \leq t \leq \mathcal{X}_{i}+\mathcal{Y}_{i}\right)$.

While preparing the initial schedule $\mathcal{S}$, we can use $P_{i}^{d}(t)$ as a benchmark to sequence the jobs on the machine. In the rest of the paper, when it is not necessary to include index $i$, we will drop it from notation $\mathcal{X}_{i}, \mathcal{Y}_{i}$ and $P_{i}^{d}(t)$. We can calculate $P^{d}(t)$ as shown in Lemma 3.1.

Lemma 3.1. Let $f_{\mathcal{X}}, F_{\mathcal{X}}, f_{\mathcal{Y}}$, and $F_{\mathcal{Y}}$ be probability density functions and distribution functions of continuous random variables $\mathcal{X}$ and $\mathcal{Y}$, respectively. Then,

$$
\begin{gathered}
P^{d}(t)=P(\mathcal{X} \leq t \leq \mathcal{X}+\mathcal{Y})=\int_{-\infty}^{t}\left(1-F_{\mathcal{Y}}(t-x)\right) \\
\cdot f_{\mathcal{X}}(x) d x=\int_{0}^{\infty}\left(F_{\mathcal{X}}(t)-F_{\mathcal{X}}(t-y)\right) f_{\mathcal{Y}}(y) d y .
\end{gathered}
$$


Proof.

$$
\begin{aligned}
P(\mathcal{X} \leq t \leq \mathcal{X}+\mathcal{Y}) & =\int_{-\infty}^{t} P(\mathcal{X} \leq t \leq \mathcal{X}+\mathcal{Y} \mid \mathcal{X}=x) \cdot f_{\mathcal{X}}(x) d x \\
& =\int_{-\infty}^{t} P(\mathcal{Y} \geq t-x) \cdot f_{\mathcal{X}}(x) d x \\
& =\int_{-\infty}^{t}\left(1-F_{\mathcal{Y}}(t-x)\right) \cdot f_{\mathcal{X}}(x) d x
\end{aligned}
$$

Similarly conditioning on $y$ immediately brings up the second equality.

Lemma 3.1 defines $P^{d}(t)$ which gives the probability that a machine will fail before or at time $t$ and will not be available at time $t$. The next property states that $P^{d}(t)$ can have a unique local maximum in the interval $[0, \infty]$.

Lemma 3.2. If $f_{x}$ is unimodal in the interval $[0, \infty]$, i.e. it has a unique local maximum in the interval $[0, \infty]$, then $P^{d}(t)$ is also unimodal in the interval $[0, \infty]$.

Proof. The first derivative of $P^{d}(t)$ is

$\frac{\partial P^{d}(t)}{\partial t}=\left(1-F_{\mathcal{Y}}(0)\right) f_{\mathcal{X}}(t)-\int_{-\infty}^{t} f_{\mathcal{Y}}(t-x) f_{\mathcal{X}}(x) d x$.

The second term in the derivative expression is an integral of the multiplication of non-negative functions. Hence, the term is nonnegative and increasing in given interval. Since the first term is unimodal by definition, the derivative of $P^{d}(t)$ can take the value zero only at a single point in the interval and hence $P^{d}(t)$ is unimodal.

Lemma 3.2 implies that the downtime probability is first increasing and then decreasing. So, there is a time point where the downtime probability is at its maximum. Lemma 3.2 is quite important in designing the probabilistic sequencing algorithm which will be discussed in Section 3.4. Lemma 3.2 implies that $P^{d}(t)$ takes its minimum value at one of the boundary points of operating interval $\left[0, D_{i}\right]$. If $D_{i}$ is large enough such that the $P^{d}(t)$ is minimized in the interior of $\left[0, D_{i}\right]$, then the jobs which are not flexible to reschedule should be scheduled close to the boundary points.

For the experimental study given in Section 4, we considered four probability distribution pairs for failure and repair times, which are Normal-Normal (Norm-Norm), Triangular-Normal (Tri-Norm), Exponential-Normal (Exp-Norm), and ExponentialExponential (Exp-Exp) distributions. The first distribution of each pair is the failure time distribution and the second distribution is for the repair time. In each case, density function for the failure time distribution is unimodal in the considered interval, so they satisfy the condition of Lemma 3.2 and hence $P^{d}(t)$ is a unimodal function in each case. We present the derivation of $P^{d}(t)$ for each distribution pair in Appendix. Next, we define the flexibility measures which we use to assess the flexibility of each job with respect to considered rescheduling problem.

\subsection{Flexibility measures}

In our anticipative approach, the goal is to prepare an initial schedule, i.e. find a job sequence on each machine, which is flexible against machine breakdowns with respect to rescheduling cost. Thus, as the third step of our approach, we introduce new flexibility measures. We consider a rescheduling problem in which the objective is to minimize the rescheduling cost subject to a given match-up time. We define a "flexible" schedule as the one which can be repaired at minimum possible manufacturing cost increase after a machine breakdown. In order to find a job sequence on a machine, it is crucial to use a measure which ranks jobs by their ability to absorb a disruption at minimum cost. Below we list the measures which could affect our anticipative scheduling decisions and explain why each measure is critical for the considered rescheduling problem.

Processing time $\left(p_{j}\right)$ : is the processing time of a job $j$ in the initial schedule, i.e. $p_{j}=p_{i j}^{u}-y_{i j}^{\mathcal{S}}$ where $i$ is the machine that job $j$ is assigned in $\mathcal{A}$. Processing time is critical for the rescheduling problem since placing shorter jobs around a downtime period could allow to distribute the required compression to more jobs and hence improve cost performance since the cost functions are convex.

Compressibility $\left(w_{j}\right)$ : is the available amount of further compression for job $j$ on its current machine in $\mathcal{A}$. It is assigned to $w_{j}=\left\{u_{i j}-y_{i j}^{\mathcal{S}}\right\}$ where $i$ is job $j$ 's current machine. Compressibility of a job is the ability to occupy less capacity on a machine and hence gives us a measure on how much of the downtime it can absorb after a disruption. The higher the compressibility of jobs in the downtime zone, it is possible to achieve the smaller match-up times.

Second derivative of compression cost function $\left(f_{j}^{\prime \prime}\right)$ : Suppose that job $j$ is assigned to machine $i$ in $\mathcal{A}$ and selected optimal compression level is $y_{i j}^{\mathcal{S}}$, then $f_{j}^{\prime \prime}=\partial^{2} f\left(y_{i j}^{\mathcal{S}}\right) / \partial y_{i j}^{2}$. By definition, the second derivative of a function gives the change rate of the first derivative at a point where it is evaluated. Lemma 3.3 gives an optimality property for the problem MJA for the compression levels on the processing times and first derivatives of cost functions for the jobs assigned on the same machine.

Lemma 3.3. Let $y_{i j_{1}}^{*}$ and $y_{i j_{2}}^{*}$ be the optimal compression levels for jobs $j_{1}$ and $j_{2}$ assigned on machine $i$ in the optimal solution to MJA. Let the corresponding first derivatives of the compression cost functions be $\lambda_{j_{1}}=\left(\partial f_{i j_{1}} / \partial y_{i j_{1}}\right)\left(y_{i j_{1}}^{*}\right)$ and $\lambda_{j_{2}}=\left(\partial f_{i j_{2}} / \partial y_{i j_{2}}\right)\left(y_{i j_{2}}^{*}\right)$. Then, one of the following statements holds:

(i) $\lambda_{j_{1}}=\lambda_{j_{2}}$ and $0 \leq y_{i j_{1}}^{*} \leq u_{i j_{1}}$ and $0 \leq y_{i j_{2}}^{*} \leq u_{i j_{2}}$;

(ii) $\lambda_{j_{1}}<\lambda_{j_{2}}$ and $y_{i j_{1}}^{*}=u_{i j_{1}}$ and $0 \leq y_{i j_{2}}^{*} \leq u_{i j_{2}}$;

(iii) $\lambda_{j_{2}}<\lambda_{j_{1}}$ and $0 \leq y_{i j_{1}}^{*} \leq u_{i j_{1}}$ and $y_{i j_{2}}^{*}=u_{i j_{2}}$.

Proof. It can easily be observed that a solution, in which there exists two jobs which violate the lemma, can be improved by changing the compression levels of the jobs.

Lemma 3.3 states that, in $\mathcal{A}$, on each machine the first derivatives of compression cost functions of jobs at optimal compression levels are equal. Lemma 3.3 shows that an exception can be fully compressed jobs $\left(y_{i j}^{*}=u_{i j}\right)$. This implies that in $\mathcal{A}$ marginal compression cost values are equal for the jobs assigned to the same machine. Then, it is intuitive to look at the second derivatives of the cost functions to estimate the cost function behaviors. If $f_{j_{1}}^{\prime \prime}>f_{j_{2}}^{\prime \prime}$, then we can say that the increase rate of the derivative of job $j_{1}$ is higher than $j_{2}$ and hence we can expect the cost increase rate of job $j_{1}$ to be higher around the optimal solution. As a result, in order to minimize the compression cost required to absorb a downtime, we may place the jobs with smaller second derivatives to the regions where a possible downtime is more likely to occur.

$\operatorname{Delta}\left(\Delta_{i}\right): \Delta$ is the average slope of the compression cost function of job $j$ on machine $i$ given in $\mathcal{A}$ in the interval $\left[y_{i j}^{\mathcal{S}}, u_{i j}\right]$. We calculate this flexibility measure as

$\Delta=\frac{f\left(u_{i j}\right)-f\left(y_{i j}^{\mathcal{S}}\right)}{u_{i j}-y_{i j}^{\mathcal{S}}}$.

$\Delta$ is another measure which provides us information on the behavior of compression cost function. Different than $f^{\prime \prime}, \Delta$ not only considers a local behavior but it looks ahead to see what happens if the job is fully compressed. When sequencing the jobs 
on a machine, it would be better to place jobs with smaller $\Delta$ values to the time periods with higher probability of downtime.

When rescheduling jobs, we may need to reallocate some jobs to other machines in order to minimize the rescheduling cost. Usually, it is more likely to move jobs from the disrupted machine to other machines. Then, estimating the cost change that will occur when we move a job from its original machine to another machine can also help to rank the flexibility of the job. The cost change lower bound for moving job $j$ from machine $i_{1}$ to machine $i_{2}$ can be calculated as below:

Lemma 3.4. For a given machine-job assignment $\mathcal{A}$, let $\lambda_{i_{1}}$ and $\lambda_{i_{2}}$ be the derivative values of compression cost functions of jobs on machines $i_{1}$ and $i_{2}$, respectively, and $y_{i_{1} j}^{\mathcal{A}}$ be the compression of job $j$ on machine $i_{1}$. Then, a lower bound for the cost change that will result by moving job $j$ from machine $i_{1}$ to $i_{2}$ is as stated below:

$$
\begin{aligned}
& L B\left(j:\left(i_{1} \rightarrow i_{2}\right)\right)=-\lambda_{i_{1}}\left(p_{i_{1} j}-y_{i_{1} j}^{\mathcal{A}}\right)-c_{i_{1} j} \\
& \quad-f_{i_{1} j}\left(y_{i_{1} j}^{\mathcal{A}}\right)+c_{i_{2} j}+f_{i_{2} j}\left(\hat{y}_{i_{2} j}\right)+\lambda_{i_{2}}\left(p_{i_{2} j}-\hat{y}_{i_{2} j}\right), \\
& \text { where } \hat{y}_{i_{2} j}=\min \left(\left(\partial f_{i_{2} j} / \partial y_{i_{2} j}\right)^{-1}\left(\lambda_{i_{2}}\right), u_{i_{2} j}\right) .
\end{aligned}
$$

For the proof of Lemma 3.4, we refer the reader to Gürel and Aktürk [8]. Given the cost change lower bounds for moving a job from its current machine to the other machines, we can define the following flexibility measure for each job.

Minimum re-allocation cost lower Bound $\left(L B_{j}\right)$ : The minimum cost change lower bound for moving job $j$ from its initially assigned machine in $\mathcal{A}$ to the some other machine can be calculated as follows:

$L B_{j}=\min _{i_{2}}\left\{L B\left(j:\left(i_{1} \rightarrow i_{2}\right)\right): \forall i_{2}, i_{2} \neq i_{1}\right\}$,

where $i_{1}$ is the initially assigned machine of job $j$. This measure can be used such that we can place the jobs with smaller reallocation cost to the time periods where the downtime probability is higher.

We have defined a set of measures which may help to make sequencing decisions. We can also combine these measures to form a new flexibility measure as defined below:

Definition 1. A flexibility measure $F$ is a multiplication of integer powers of several flexibility factors. More formally,

$F_{j}=\left(p_{j}\right)^{\alpha_{1}} \times\left(w_{j}\right)^{\alpha_{2}} \times\left(f_{j}^{\prime \prime}\right)^{\alpha_{3}} \times\left(\Delta_{j}\right)^{\alpha_{4}} \times\left(L B_{j}\right)^{\alpha_{5}}$,

where $\alpha_{k} \in \mathbb{Z}$.

In order to clarify how these flexibility factors could be used as a sequencing rule, $\max \{1 / p\}$ corresponds to the shortest processing time (SPT) rule, whereas $\max \left\{w^{2} / p \cdot \Delta \cdot L B\right\}$ is a composite rule that combines four of them into a single sequencing rule.

Next, we give an algorithm which schedules the jobs on their assigned machines by considering the downtime probability $P^{d}(t)$ function of each machine and the flexibility measure $F_{j}$ for each job.

\subsection{Probabilistic sequencing algorithm}

Probabilistic sequencing algorithm finds a job sequence on a given machine by considering the flexibility measures of the jobs and the downtime probability function for the machine. The goal is to place the jobs with maximum flexibility to the positions with the maximum probability of downtime. The interval considered for machine $i$ in this algorithm is $\left[0, D_{i}\right]$. Let $F_{j}$ be the flexibility measure of job $j . F_{j}$ can be easily computed for all jobs. In the first step of the algorithm, we order the jobs in $J_{i}$ in ascending order of $F_{j}$. Then, starting with the first job in the list, the algorithm places each job into the schedule one by one. For the first job, say job $j$, the available interval is $\left[0, D_{i}\right]$. Proposed algorithm evaluates two alternatives. The first one is placing the job at the beginning of the available interval. The second alternative is placing it at the end. The algorithm checks the downtime probability at the mid-point of the job in both cases, i.e. checks $P^{d}\left(p_{j} / 2\right)$ and $P^{d}\left(D_{i}-p_{j} / 2\right)$. If the first probability is less, then the algorithm places the job at $\left[0, p_{j}\right]$. Else, the job is placed at $\left[D_{i}-p_{j}, D_{i}\right]$. Then, the algorithm updates the available interval and takes the next job from the list.

We check only the boundaries of the available interval, since we know from Lemma 3.2 that if $f_{\mathcal{X}}$ is a unimodal function then the probability function $P^{d}(t)$ is also unimodal in the interval $\left[0, D_{i}\right]$ for machine $i$. $P^{d}(t)$ being unimodal implies that the minimum downtime probability in the interval is found at one of the boundary points of the interval. Therefore, the algorithm tries to place the least flexible jobs first to the start or end points of the interval, i.e. to the position with minimum downtime probability. We give the step by step definition in Algorithm 2 .

\section{Algorithm 2. Probabilistic Sequencing Algorithm.}

Require: Machine $i$ with $P^{d}(t)$ and available interval $\left[t_{s}, t_{e}\right]$.

Require: Set of jobs $J_{i}$ with $F_{j}$ and $p_{j}$ for each $j \in J_{i}$. Initialize: Order the jobs in $J_{i}$ in ascending order of $F_{j}{ }^{\prime} \mathrm{s}$; Initialize: $t_{s}=0$ and $t_{e}=D_{i}$;

for each job $j \in J_{i}$ do

if $P^{d}\left(t_{s}+p_{j} / 2\right) \leq P^{d}\left(t_{e}-p_{j} / 2\right)$ then

Schedule job $j$ at $\left[t_{s}, t_{s}+p_{j}\right]$.

$t_{s}=t_{s}+p_{j}$.

else

Schedule job $j$ at $\left[t_{e}-p_{j}, t_{e}\right]$.

$t_{e}=t_{e}-p_{j}$.

In the following, we provide a numerical example which illustrates Algorithm 1.

\begin{tabular}{|c|c|c|c|c|c|c|c|c|c|c|c|c|c|c|c|c|}
\hline \multirow[t]{2}{*}{ Machine } & \multirow[t]{2}{*}{ Constant } & \multicolumn{15}{|c|}{ Jobs } \\
\hline & & 1 & 2 & 3 & 4 & 5 & 6 & 7 & 8 & 9 & 10 & 11 & 12 & 13 & 14 & 15 \\
\hline \multirow[t]{5}{*}{1} & $c$ & 3.2 & 2.2 & 2.9 & 3 & 3.4 & 3.9 & 3.1 & 5.1 & 2.5 & 3.2 & 3.8 & 2.5 & 2 & 2.8 & 3.6 \\
\hline & $k$ & 1 & 1.3 & 2.2 & 2.2 & 1.5 & 2.8 & 1.1 & 1.4 & 2.4 & 1.3 & 2.6 & 2.3 & 2.6 & 1.5 & 2.6 \\
\hline & $a / b$ & 2.9 & 1.6 & 2.4 & 2.8 & 3.1 & 3.1 & 1.6 & 1.6 & 2.9 & 2.2 & 2 & 3.1 & 2.3 & 1.9 & 2.3 \\
\hline & $p_{u}$ & 2.6 & 1.4 & 1.1 & 2 & 2.7 & 2.1 & 2.7 & 2.7 & 1.3 & 1.2 & 2.3 & 1.1 & 1.6 & 2.7 & 1.3 \\
\hline & $u$ & 1.7 & 0.9 & 0.9 & 1.6 & 2.1 & 1.5 & 1.9 & 2 & 0.8 & 0.8 & 1.5 & 0.9 & 1.2 & 1.5 & 0.7 \\
\hline \multirow[t]{5}{*}{2} & $c$ & 3.5 & 3 & 5.9 & 3.4 & 4.9 & 5.2 & 5.9 & 2.3 & 5.8 & 3.5 & 2.3 & 4.4 & 3.7 & 3.6 & 4.5 \\
\hline & $k$ & 1.2 & 2.2 & 1.4 & 2.3 & 1.4 & 2.7 & 1.4 & 2.1 & 2.6 & 1.7 & 2 & 1.1 & 2.6 & 2.8 & 1.4 \\
\hline & $a / b$ & 1.2 & 2 & 1.5 & 3.1 & 1.5 & 1.6 & 1.7 & 2 & 2.7 & 1.7 & 2.5 & 2.7 & 1.7 & 2.1 & 1.9 \\
\hline & $p_{u}$ & 1.2 & 2.6 & 1.6 & 1.1 & 2.9 & 2.9 & 3 & 2.8 & 2.6 & 1.1 & 1.9 & 2.1 & 2.2 & 2 & 2.4 \\
\hline & $u$ & 0.9 & 1.4 & 1.4 & 0.6 & 2.2 & 1.7 & 1.7 & 2 & 1.6 & 0.6 & 1.4 & 1.4 & 2 & 1.3 & 1.4 \\
\hline
\end{tabular}

Table 1

Numerical example data. 
Table 2

Machine-job assignment and flexibility measures.

\begin{tabular}{|c|c|c|c|c|c|c|c|}
\hline \multirow[t]{2}{*}{ Machine } & \multirow[t]{2}{*}{ Job } & \multicolumn{6}{|c|}{ Flexibility measures } \\
\hline & & $p$ & $w$ & $f^{\prime \prime}$ & $\Delta$ & $L B$ & $\frac{w^{2}}{p \Delta L B}\left(10^{-3}\right)$ \\
\hline \multirow[t]{9}{*}{1} & 2 & 0.50 & 0.0 & 1.30 & 1.95 & 4.19 & 0.0 \\
\hline & 7 & 0.80 & 0.0 & 0.82 & 2.59 & 5.56 & 0.0 \\
\hline & 15 & 0.72 & 0.12 & 6.59 & 3.34 & 4.98 & 1.24 \\
\hline & 9 & 0.67 & 0.17 & 8.78 & 3.71 & 7.52 & 1.48 \\
\hline & 6 & 1.50 & 0.90 & 10.32 & 10.27 & 8.64 & 6.12 \\
\hline & 3 & 0.44 & 0.24 & 6.25 & 3.73 & 5.32 & 6.74 \\
\hline & 12 & 0.45 & 0.25 & 9.40 & 4.24 & 4.70 & 6.77 \\
\hline & 5 & 1.90 & 1.30 & 7.67 & 10.95 & 9.72 & 8.34 \\
\hline & 13 & 1.02 & 0.62 & 6.59 & 5.17 & 6.58 & 11.14 \\
\hline \multirow[t]{6}{*}{2} & 4 & 0.67 & 0.17 & 5.96 & 1.80 & 4.86 & 4.81 \\
\hline & 11 & 1.51 & 1.01 & 4.70 & 4.41 & 9.07 & 16.84 \\
\hline & 14 & 1.76 & 1.06 & 5.61 & 4.45 & 7.70 & 18.61 \\
\hline & 8 & 2.51 & 1.71 & 4.20 & 4.81 & 11.89 & 20.32 \\
\hline & 10 & 0.81 & 0.31 & 2.92 & 1.64 & 2.44 & 29.0 \\
\hline & 11 & 0.75 & 0.45 & 0.55 & 1.33 & 5.85 & 34.91 \\
\hline
\end{tabular}

Table 3

Implementation of Algorithm 2

\begin{tabular}{|c|c|c|c|c|c|c|c|}
\hline Machine & Job & $t_{s}$ & $P^{d}\left(t_{s}+p_{j} / 2\right)$ & $t_{e}$ & $P^{d}\left(t_{e}-p_{j} / 2\right)$ & Start time & End time \\
\hline \multirow[t]{9}{*}{1} & 2 & 0.00 & 0.10 & 8.00 & 0.02 & 7.50 & 8.00 \\
\hline & 7 & 0.00 & 0.15 & 7.50 & 0.03 & 6.70 & 7.50 \\
\hline & 15 & 0.00 & 0.14 & 6.70 & 0.04 & 5.98 & 6.70 \\
\hline & 9 & 0.00 & 0.13 & 5.98 & 0.06 & 5.31 & 5.98 \\
\hline & 6 & 0.00 & 0.22 & 5.31 & 0.09 & 3.81 & 5.31 \\
\hline & 3 & 0.00 & 0.09 & 3.81 & 0.14 & 0.00 & 0.44 \\
\hline & 12 & 0.44 & 0.20 & 3.81 & 0.14 & 3.36 & 3.81 \\
\hline & 5 & 0.44 & 0.25 & 3.36 & 0.21 & 1.47 & 3.36 \\
\hline & 13 & 0.44 & 0.24 & 1.47 & 0.24 & 0.44 & 1.47 \\
\hline \multirow[t]{6}{*}{2} & 4 & 0.00 & 0.13 & 8.00 & 0.02 & 7.33 & 8.00 \\
\hline & 11 & 0.00 & 0.22 & 7.33 & 0.04 & 5.82 & 7.33 \\
\hline & 14 & 0.00 & 0.23 & 5.82 & 0.08 & 4.07 & 5.82 \\
\hline & 8 & 0.00 & 0.25 & 4.07 & 0.19 & 1.56 & 4.07 \\
\hline & 10 & 0.00 & 0.15 & 1.56 & 0.25 & 0.00 & 0.81 \\
\hline & 1 & 0.81 & 0.25 & 1.56 & 0.25 & 0.81 & 1.56 \\
\hline
\end{tabular}

\subsection{Numerical example}

We consider a numerical example with $n=15$ and $m=2$. Table 1 gives cost function coefficients, processing time upper bound and compression upper bound for each machine-job pair. As data shows, machines are non-identical and each job has different cost function, different processing time upper bound and compression upper bound.

In the first step of Algorithm 1, machine-job assignment (MJA) problem is solved to find minimum cost machine-job assignment for $D[i]=8.0, i=1,2$. An optimal machine-job assignment is given in Table 2. In the second step, the downtime probability function is calculated for each machine. In this example, we assume an exponential failure time and an exponential repair time and take $\lambda_{\mathcal{X}}=0.5$ and $\lambda_{y}=1.0$ for both machines. Consequently, $P^{d}(t)=e^{-0.5 t}-e^{-t}$ as discussed in the Appendix. In the third step, flexibility measures are calculated for each machine-job pair. At this step, a combined flexibility measure $F_{j}=w^{2} / p \Delta L B$ is used as an example, and the corresponding values for each job on each machine are given in Table 2. $F_{j}$ values will be used in sequencing decisions in Step 4.

In Step 4 of Algorithm 1, Probabilistic Sequencing Algorithm (Algorithm 2) is implemented. Algorithm 2 is executed once for
Gantt Chart 1. Anticipative schedule given by Algorithm 2

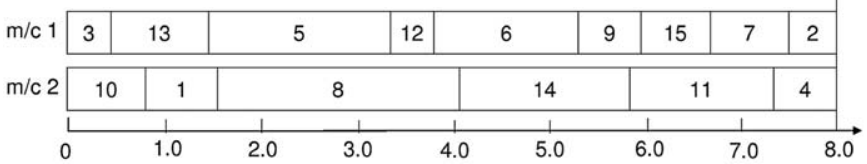

Gantt Chart 2. Schedule given by the SPT rule

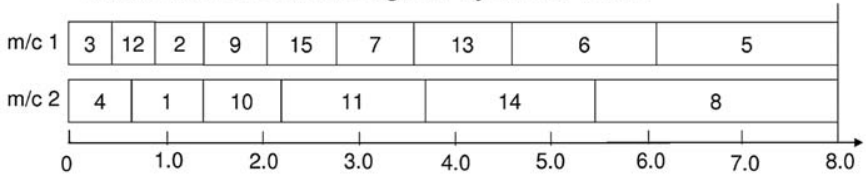

Fig. 1. Different schedules generated by Algorithm 2 .

each machine. Given machine-job assignments in Table 2, in the first step all jobs are sorted in ascending order of $w^{2} / p \Delta L B$ for each machine. Then, in the next step of the algorithm, each job in the list is scheduled at the beginning or at the end of available scheduling interval by considering $P^{d}(t)$ levels at both ends of the interval. Calculations of $P^{d}\left(t_{s}+p_{j} / 2\right), P^{d}\left(t_{e}-p_{j} / 2\right)$ and scheduled start and end times of all jobs are given in Table 3 .

In the given solution of MJA, processing times of jobs 2 and 7 on machine 1 are no longer compressible as they are already compressed at full. Thus, flexibility of these two jobs are measured to be zero. As downtime probability function is at its minimum at the end of scheduling interval, Algorithm 2 schedules jobs 2 and 7 to the end of the interval on machine 1 . On the other hand, since jobs 5 and 13 have the highest two flexibility measure values, Algorithm 2 schedules them at the time period with the highest down time probability for machine 1 .

Algorithm 1 , using $F_{j}=w^{2} / p \Delta L B$ as the flexibility measure, achieves the schedule given in Gantt Chart 1 in Fig. 1. If the SPT rule is used to sequence jobs, then the schedule represented by Gantt Chart 2 in Fig. 1 is obtained. If a breakdown occurs on one of the machines, schedules by Algorithm 2 and by the SPT rule would absorb caused disruption at different rescheduling costs. In the next section, we compare rescheduling costs of schedules achieved by Algorithm 2 by using different flexibility measures against the schedules obtained by the SPT rule.

\section{Computational study}

In the computational study, we tested the performance of Algorithm 2 using alternative flexibility measures $F_{j}$ described in Section 3.3. We compared rescheduling performance on the initial schedules achieved by Algorithm 2 against the performance of initial schedules achieved by using the SPT rule. Adiri et al. [15] consider, for the first time, the flow-time scheduling problem when the machine faces breakdowns at stochastic time epochs, the repair time is stochastic, but the processing times are constant. They prove that the problem is NP-hard and show that the SPT rule minimizes the expected total flow time if the time to breakdown is exponentially distributed. Lee and Liman [16] study the deterministic equivalent of this problem in the context of a single scheduled maintenance and find a tight performance bound of $9 / 7$ for the SPT rule.

In the test problems, number of jobs is $n=100$, and number of machines is $m=3$ initially. We generated manufacturing cost $\left(c_{i j}\right)$ for each machine-job pair randomly from Uniform[2.0,6.0]. We generated $k_{i j}$ coefficient of the compression cost function $\left(f_{i j}\left(y_{i j}\right)=k_{i j} y_{i j}^{a_{i j} / b_{i j}}\right)$ from Uniform[1.0,3.0] and $a_{i j} / b_{i j}$ from Uniform $[1.1,3.1]$. We generated processing time upper bound $p_{i j}^{u}$ from Uniform [1.0,5.0]. In practice, one can expect a correlation between processing time upper bound and the maximum 
compressibility at least due to the fact that processing time upper bound is an upper bound for the maximum compressibility. Thus, we generated the compression bound $u_{i j}$ from $p_{i j}^{u} \times$ Uniform $[0.5$, 0.9]. We set the machining capacity of each machine as below:

$D_{i}=0.2 \times \frac{\sum_{i=1}^{m} \sum_{j=1}^{n} p_{i j}^{u}}{m}$.

In order to construct initial schedules, we first solved the machine-job assignment problem given in Section 3.1. We sequenced the jobs assigned on each machine by using Algorithm 2 which employed each of the following proposed flexibility measures: $1 / p \Delta, 1 / f^{\prime \prime} L B, 1 / p f^{\prime \prime} L B, 1 / p \Delta L B, w / p, w / p f^{\prime \prime} L B$, $w / p \Delta L B$ and $w^{2} / p \Delta L B$. We also formed an initial schedule by using the SPT rule on each machine, which gives the minimum total completion time.

For $\mathcal{X}_{i}$ and $\mathcal{Y}_{i}$, we used four different distribution pairs consisting of Norm-Norm, Exp-Exp, Exp-Norm, and Tri-Norm. Having formed initial schedules, we randomly selected a machine to fail. We generated a failure time, $\mathcal{X}_{i}$, and a repair time $\mathcal{Y}_{i}$ for each machine $i$.

In failure time distribution, mean time to fail is $\mathrm{MTTF}=0.3 \cdot D[i]$. For exponential distribution, $\lambda=1 / \mathrm{MTTF}$. For normal distribution, standard deviation is generated by $\sigma=0.5$. MTTF . $Z$ where $Z \sim$ Uniform $[0,1]$. We used $0, D[i]$ and MTTF as the parameters of a triangular distribution. In repair time distribution, we used two different levels of mean time to repair, denoted as MTTR. For all distributions except exponential distribution, we used $\operatorname{MTTR}=0.1 \cdot D[i]$ and $\operatorname{MTTR}=0.15 \cdot D[i]$. For exponential distribution, we adjusted MTTR and MTTF parameters in order to avoid high variability, since high variability leads to long failure

Table 4

Mean rescheduling cost performance $R(\%)$ for the Norm-Norm case.

\begin{tabular}{llllllll}
\hline MTTR & $\beta$ & \multicolumn{7}{l}{ Flexibility measures } \\
\cline { 3 - 8 } & & $\frac{w^{2}}{p \Delta L B}$ & $\frac{1}{f^{\prime \prime} L B}$ & $\frac{w}{p f^{\prime \prime} L B}$ & $\frac{1}{p f^{\prime \prime} L B}$ & $\frac{w}{p \Delta L B}$ & $\frac{1}{p 4}$ \\
\hline \multirow{2}{*}{ Low } & 0.1 & 40.5 & 41.8 & 38.8 & 22.6 & 27.5 & 19.6 \\
& 0.15 & 33.4 & 33.5 & 23.8 & 28.1 & 18.5 & 20.2 \\
& 0.20 & 31.3 & 29.7 & 20.8 & 25.0 & 16.5 & 20.3 \\
& 0.25 & 28.7 & 26.2 & 16.5 & 24.0 & 14.4 & 19.7 \\
High & Total & 33.5 & 32.8 & 25.0 & 24.9 & 19.2 & 20.4 \\
& 0.1 & 38.8 & 31.9 & 34.4 & 24.5 & 28.7 & 15.2 \\
& 0.15 & 29.2 & 27.7 & 26.3 & 23.6 & 24.4 & 15.8 \\
& 0.20 & 26.7 & 23.7 & 22.7 & 18.3 & 23.5 & 18.3 \\
& 0.25 & 19.3 & 19.0 & 14.7 & 13.2 & 15.0 & 11.3 \\
Total & Total & 28.5 & 25.6 & 24.5 & 19.9 & 22.9 & 15.1 \\
& & 31.0 & 29.2 & 24.8 & 22.4 & 21.1 & 17.8 \\
\hline
\end{tabular}

or repair times which would result infeasible rescheduling problems.

For each $\mathcal{S}$, we first solved the minimum match-up time problem to find $\left(\bar{W}_{\min }\right)$. Then, for $\bar{W}=\bar{W}_{\min }+\beta \times\left(D[i]-\bar{W}_{\min }\right)$ we solved the RCM problem for four different levels of $\beta=0.1,0.15,0.2,0.25$, so that we could generate alternative time/cost trade-offs. We took 10 replications for each setting. All experiments were performed using ILOG Cplex Version 11.2 on a $2 \times 2.83 \mathrm{GHz}$ Intel Xeon CPU and $8 \mathrm{~GB}$ memory workstation HP with the operating system Ubuntu 8.04.

For each instance, we calculated a relative difference between rescheduling costs of schedules achieved by Algorithm 2 and SPT rule. We define the relative difference $R$ as follows:

$R=100 \times \frac{\operatorname{Cost}_{S P T}-\operatorname{Cost}_{F}}{\operatorname{Cost}_{F}}$

in which Cost $_{S P T}$ is the rescheduling cost of an SPT schedule for the considered failure-repair times and match-up time. $\operatorname{Cost}_{F}$ is the rescheduling cost of a schedule achieved by Algorithm 2 using flexibility measure $F$.

Table 4 shows average $R$ results for Norm-Norm case. Flexibility measure $w^{2} / p \Delta L B$ achieves the best cost performance against the SPT rule with an average relative difference of $31 \%$. Results in Table 4 show that including cost function related flexibility measures such as $f^{\prime \prime}, \Delta$ and $L B$ in a sequencing rule significantly improves rescheduling performance. The second best flexibility measure is $1 / f^{\prime \prime} L B$. Comparing its performance with performance of $1 / p f^{\prime \prime} L B$, we can say that for Norm-Norm case including $p$ in a flexibility measure did not improve rescheduling performance. On the other hand, including $w$ seems to enhance

Table 6

Mean rescheduling cost performance $R(\%)$ for the Exp-Exp case.

\begin{tabular}{|c|c|c|c|c|c|c|}
\hline \multirow[t]{2}{*}{ MTTR } & \multirow[t]{2}{*}{$\beta$} & \multicolumn{5}{|c|}{ Flexibility measures } \\
\hline & & $\frac{1}{p f^{\prime \prime} L B}$ & $\frac{w}{p f^{\prime \prime} L B}$ & $\frac{1}{p \Delta L B}$ & $\frac{w}{p \Delta L B}$ & $\frac{1}{p \Delta}$ \\
\hline \multirow[t]{5}{*}{ Low } & 0.1 & 14.4 & 4.6 & 3.1 & 3.0 & 17.6 \\
\hline & 0.15 & 14.1 & 5.9 & 10.8 & 2.5 & 21.5 \\
\hline & 0.2 & 11.3 & 5.8 & 6.6 & 4.1 & 19.3 \\
\hline & 0.25 & 9.4 & 5.6 & 7.8 & 3.2 & 20.1 \\
\hline & Total & 12.3 & 5.5 & 7.1 & 3.2 & 19.6 \\
\hline \multirow[t]{5}{*}{ High } & 0.1 & 18.1 & 24.2 & 19.9 & 22.7 & 2.1 \\
\hline & 0.15 & 17.9 & 19.0 & 17.2 & 19.1 & 2.6 \\
\hline & 0.20 & 17.9 & 17.6 & 16.2 & 19.1 & 0.7 \\
\hline & 0.25 & 12.9 & 15.6 & 12.2 & 13.2 & -3.6 \\
\hline & Total & 16.7 & 19.1 & 16.4 & 18.5 & 0.4 \\
\hline Total & & 14.6 & 12.6 & 12.0 & 11.2 & 9.6 \\
\hline
\end{tabular}

Table 5

Confidence intervals for the mean $R$ and number of times best for the Norm-Norm case.

\begin{tabular}{|c|c|c|c|c|c|}
\hline \multirow[t]{2}{*}{ Flexibility measures } & \multicolumn{2}{|c|}{$95 \% \mathrm{CI}$ on mean $R$} & \multicolumn{3}{|c|}{ \# of times best } \\
\hline & Lower bound & Upper bound & MTTR low & MTTR high & Total \\
\hline$w^{2}$ & 24.5 & 37.5 & 35 & 33 & 68 \\
\hline \multicolumn{6}{|l|}{$\overline{p \Delta L B}$} \\
\hline 1 & 23.0 & 35.4 & 35 & 32 & 67 \\
\hline \multicolumn{6}{|l|}{$\overline{f^{\prime \prime} L B}$} \\
\hline$w$ & 17.8 & 31.7 & 31 & 35 & 66 \\
\hline \multicolumn{6}{|l|}{$\overline{p f^{\prime \prime} L B}$} \\
\hline 1 & 16.0 & 28.8 & 28 & 25 & 53 \\
\hline \multicolumn{6}{|l|}{$\overline{p f^{\prime \prime} L B}$} \\
\hline$w$ & 15.3 & 26.8 & 31 & 36 & 67 \\
\hline \multicolumn{6}{|l|}{$\overline{p \Delta L B}$} \\
\hline 1 & 12.3 & 23.2 & 31 & 29 & 60 \\
\hline$\overline{p \Delta}$ & & & & & \\
\hline
\end{tabular}


rescheduling performance as we compare $w^{2} / p \Delta L B$ and $w / p \Delta L B$. As discussed in Section 3.3, $w$ measures the available amount of compression on a job and hence it is important in solving rescheduling problems.

From Table 4, it can be observed that as match-up time level increases, average value of $R$ is more likely to decrease. This means as we allow distributing the effect of a disruption to a larger portion of initial schedule, we can expect that the gain to be achieved by considering flexibility of jobs declines. In other words, as the match-up time level is decreased, it becomes critical to place more flexible jobs around downtime period. Similarly, proposed sequencing rules are more likely to perform better when MTTR is low. Low MTTR is the case in which we can expect to have less number of jobs in a rescheduling problem. Hence, rescheduling performance is more sensitive to the set of jobs to be rescheduled. We observe that proposed flexibility measures achieve better results in this case.

For the same flexibility measures, first two columns of Table 5 gives 95\% confidence interval bounds for the average value of $R$. Given bounds clearly indicate that they are significantly better than the SPT rule in achieving lower rescheduling costs. The highest lower bound for a confidence interval is achieved by the measure $w^{2} / p \Delta L B$ which is $24.5 \%$. In the same table, we also report number of times Algorithm 2 achieve better rescheduling

Table 7

Confidence intervals for the mean $R$ and number of times best for the Exp-Exp case.

\begin{tabular}{|c|c|c|c|c|c|}
\hline \multirow[t]{2}{*}{ Flexibility measures } & \multicolumn{2}{|c|}{$95 \% \mathrm{CI}$ on mean $R$} & \multicolumn{3}{|c|}{ \# of times best } \\
\hline & Lower bound & Upper bound & MTTR low & MTTR high & Total \\
\hline$\frac{1}{p f^{\prime \prime} L B}$ & 8.6 & 20.7 & 29 & 34 & 63 \\
\hline$\frac{w}{p f^{\prime \prime} L B}$ & 7.7 & 17.5 & 30 & 35 & 65 \\
\hline$\frac{1}{p \Delta L B}$ & 6.2 & 17.8 & 24 & 30 & 54 \\
\hline$\frac{w}{p \Delta L B}$ & 5.6 & 16.8 & 24 & 36 & 60 \\
\hline$\frac{1}{p \Delta}$ & 1.8 & 17.3 & 26 & 21 & 47 \\
\hline
\end{tabular}

Table 8

Mean rescheduling cost performance $R(\%)$ for the Exp-Norm case.

\begin{tabular}{|c|c|c|c|c|c|c|}
\hline \multirow[t]{2}{*}{ MTTR } & \multirow[t]{2}{*}{$\beta$} & \multicolumn{5}{|c|}{ Flexibility measures } \\
\hline & & $\frac{1}{f^{\prime \prime} L B}$ & $\frac{w}{p f^{\prime \prime} L B}$ & $\frac{1}{p f^{\prime \prime} L B}$ & $\frac{w}{p \Delta L B}$ & $\frac{w^{2}}{p \Delta L B}$ \\
\hline \multirow[t]{5}{*}{ Low } & 0.1 & 20.7 & 5.2 & 4.0 & 7.9 & -0.4 \\
\hline & 0.15 & 16.1 & 2.6 & 0.9 & 4.6 & -1.1 \\
\hline & 0.20 & 15.3 & 1.3 & 3.3 & 7.0 & 1.2 \\
\hline & 0.25 & 8.3 & -0.5 & -1.3 & 2.2 & -3.1 \\
\hline & Total & 15.1 & 2.2 & 1.7 & 5.4 & -0.8 \\
\hline \multirow[t]{5}{*}{ High } & 0.1 & 17.9 & 16.4 & 17.2 & 10.8 & 20.1 \\
\hline & 0.15 & 15.2 & 16.3 & 15.7 & 8.8 & 14.5 \\
\hline & 0.20 & 11.4 & 12.0 & 11.6 & 9.3 & 11.0 \\
\hline & 0.25 & 12.5 & 14.0 & 8.9 & 7.2 & 11.7 \\
\hline & Total & 14.2 & 14.7 & 13.3 & 9.0 & 14.3 \\
\hline Total & & 14.7 & 8.4 & 7.5 & 7.2 & 6.4 \\
\hline
\end{tabular}

Table 9

Confidence intervals for the mean $R$ and number of times best for the Exp-Norm case.

\begin{tabular}{|c|c|c|c|c|c|}
\hline \multirow[t]{2}{*}{ Flexibility measures } & \multicolumn{2}{|c|}{$95 \% \mathrm{CI}$ on mean $R$} & \multicolumn{3}{|c|}{ \# of times best } \\
\hline & Lower bound & Upper bound & MTTR low & MTTR high & Total \\
\hline 1 & 9.7 & 19.6 & 25 & 30 & 55 \\
\hline \multicolumn{6}{|l|}{$\overline{f^{\prime \prime} L B}$} \\
\hline$\frac{w}{w f D}$ & 3.1 & 13.7 & 13 & 34 & 47 \\
\hline \multicolumn{6}{|l|}{$\overline{p f^{\prime \prime} L B}$} \\
\hline 1 & 3.6 & 11.4 & 17 & 30 & 47 \\
\hline \multicolumn{6}{|l|}{$\overline{p f^{\prime \prime} L B}$} \\
\hline \multirow{2}{*}{\multicolumn{6}{|c|}{$\overline{p \Delta L B}$}} \\
\hline & & & & & \\
\hline$w^{2}$ & 0.6 & 12.9 & 18 & 30 & 48 \\
\hline$\overline{p \triangle L B}$ & & & & & \\
\hline
\end{tabular}


Table 10

Mean rescheduling cost performance $R(\%)$ for the Tri-Norm case.

\begin{tabular}{|c|c|c|c|c|c|c|}
\hline \multirow[t]{2}{*}{ MTTR } & \multirow[t]{2}{*}{$\beta$} & \multicolumn{5}{|c|}{ Flexibility measures } \\
\hline & & $\frac{w^{2}}{p \Delta L B}$ & $\frac{w}{p f^{\prime \prime} L B}$ & $\frac{w}{p \Delta L B}$ & $\frac{w}{p}$ & $\frac{1}{p \Delta L B}$ \\
\hline \multirow[t]{5}{*}{ Low } & 0.1 & 14.7 & 0.2 & 13.8 & 5.2 & 6.0 \\
\hline & 0.15 & 15.3 & 3.7 & 15.3 & 5.5 & 4.8 \\
\hline & 0.2 & 11.8 & 8.0 & 13.7 & 2.9 & 5.8 \\
\hline & 0.25 & 11.7 & 6.4 & 13.4 & 1.7 & 3.7 \\
\hline & Total & 13.4 & 4.6 & 10.4 & 3.8 & 5.1 \\
\hline \multirow[t]{5}{*}{ High } & 0.1 & 9.3 & 18.2 & 1.1 & 14.6 & 5.5 \\
\hline & 0.15 & 8.4 & 15.4 & 3.2 & 9.1 & 6.7 \\
\hline & 0.20 & 8.1 & 14.1 & 4.0 & 6.3 & 7.1 \\
\hline & 0.25 & 6.5 & 9.6 & 1.9 & 3.3 & 6.0 \\
\hline & Total & 8.1 & 14.3 & 2.5 & 8.3 & 6.3 \\
\hline Total & & 10.6 & 9.7 & 8.0 & 6.2 & 5.7 \\
\hline
\end{tabular}

Table 11

Confidence intervals for mean $R$ and number of times best for Tri-Norm case.

\begin{tabular}{|c|c|c|c|c|c|}
\hline \multirow[t]{2}{*}{ Flexibility measures } & \multicolumn{2}{|c|}{$95 \% \mathrm{CI}$ on mean $R$} & \multicolumn{3}{|c|}{ \# of times best } \\
\hline & Lower bound & Upper bound & MTTR low & MTTR high & Total \\
\hline$w^{2}$ & 3.6 & 17.6 & 28 & 27 & 55 \\
\hline \multicolumn{6}{|l|}{$\overline{p \Delta L B}$} \\
\hline$w$ & 4.1 & 15.3 & 22 & 34 & 56 \\
\hline \multicolumn{6}{|l|}{$\overline{p f^{\prime \prime} L B}$} \\
\hline$\frac{w}{p \Delta L B}$ & \multicolumn{4}{|c|}{$\overline{p \Delta L B}$} & 48 \\
\hline$\underline{w}$ & 1.5 & 10.9 & 19 & 23 & 42 \\
\hline \multicolumn{6}{|l|}{$\bar{p}$} \\
\hline 1 & 0.8 & 10.7 & 26 & 22 & 48 \\
\hline$\overline{p \Delta L B}$ & & & & & \\
\hline
\end{tabular}

cost performance than the SPT rule. The best performance is by $w^{2} / p \Delta L B$ with 68 problems out of 80 . The next best performance is by $1 / f^{\prime \prime} L B$ and $w / p \Delta L B$ with 67 out of 80 . We see that all measures perform better than the SPT sequence with the worst one performing better in 53 problems out of 80 .

Table 6 provides $R$ values for the best five flexibility measures for Exp-Exp case. Results show that $1 / p f^{\prime \prime} L B$ has achieved best average $R$ performance of $14.6 \%$. Table 6 shows that first four measures perform better when MTTR is high. On the other hand, we observe that the last measure's $(1 / p \Delta)$ performance declines as MTTR is increased. Also, $1 / p \Delta$ is the best performing measure when MTTR is low. This shows that it is essential to include the flexibility factor $L B$ (which gives the reallocation cost lower bound) in a flexibility measure if we have high repair times and hence job reallocations are more likely in rescheduling. Comparing rescheduling performance of the first measure with the second one, and similarly the third measure with the fourth one in Table 6 , we can conclude that including $w$ in a flexibility measure enhances rescheduling performance when MTTR is high.

We observe that performance of our algorithm against the SPT rule degrades slightly when exponential failure is considered. Exponential failure implies a decreasing failure rate which requires placing flexible jobs first in the sequence. When exponential failure is considered, we can expect SPT rule to form a job sequence which is quite similar to a sequence that Algorithm 2 would generate by using flexibility measure $1 / p$. Hence, we can expect SPT to perform better in exponential failure case compared to other failure distributions.

Table 7 shows that flexibility measure $1 / p f^{\prime \prime} L B$ achieves the highest lower and upper bounds for the 95\% confidence interval.
Table 7 also gives the number of times that the sequence formed by Algorithm 2 achieves a lower rescheduling cost than the SPT sequence in the number of times best section. The results show that out of 80 problems solved, the algorithm using flexibility measure $w / p f^{\prime \prime} L B$ achieved a lower rescheduling cost in 65 instances. In general, we observe that all flexibility measures perform better both in terms of average cost difference and in terms of number of times achieving lower rescheduling cost compared to the schedules formed by the SPT rule.

Table 8 shows average $R$ values for different flexibility measures tested in Exp-Norm case. Flexibility measure $1 / f^{\prime \prime} L B$ achieves the best rescheduling cost performance on the average, and it performs well for both low and high MTTR levels. We observe that performance of other measures are sensitive to the MTTR level.

In Table 9, we give the confidence intervals for the mean $R$ for Exp-Norm case. The results show that proposed sequencing algorithm significantly outperforms the SPT sequenced schedules in terms of rescheduling cost. Table 9 also includes how many times each flexibility measure achieves lower rescheduling cost compared to the SPT rule. The best measure is $1 / f^{\prime \prime} L B$ which finds a smaller cost in 55 problems out of 80 .

Table 10 shows average values of $R$ for selected flexibility measures for the Tri-Norm case. The best performing flexibility measure is $w^{2} / p \Delta L B$ with $10.6 \%$. The next best performance on the average is by $w / p f^{\prime \prime} L B$.

Despite lower average $R$ values, on the average our algorithm's performance is still significantly better than the SPT rule. Table 11 gives the 95\% confidence intervals for average $R$. Proposed sequencing algorithm using the selected flexible measures 
Table 12

Mean rescheduling cost performance $R(\%)$.

\begin{tabular}{|c|c|c|c|c|c|c|}
\hline$n-m$ & MTTR & $\beta$ & $\begin{array}{l}\text { Norm-Norm } \\
\frac{w^{2}}{p \Delta L B}\end{array}$ & $\begin{array}{l}\text { Tri-Norm } \\
\frac{w^{2}}{p \Delta L B}\end{array}$ & $\begin{array}{l}\text { Exp-Exp } \\
\frac{1}{p f^{\prime \prime} L B}\end{array}$ & $\begin{array}{l}\text { Exp-Norm } \\
\frac{1}{f^{\prime \prime} L B}\end{array}$ \\
\hline \multirow[t]{11}{*}{$150-5$} & \multirow[t]{5}{*}{ Low } & 0.1 & 11.6 & 7.0 & 17.4 & 10.7 \\
\hline & & 0.15 & 9.9 & 7.4 & 20.5 & 12.0 \\
\hline & & 0.2 & 11.1 & 4.6 & 17.9 & 9.7 \\
\hline & & 0.25 & 10.8 & 1.8 & 14.7 & 7.7 \\
\hline & & Total & 10.9 & 5.2 & 17.6 & 10.0 \\
\hline & \multirow[t]{5}{*}{ High } & 0.1 & 14.7 & 12.0 & 4.5 & 16.3 \\
\hline & & 0.15 & 10.1 & 10.9 & 12.1 & 12.2 \\
\hline & & 0.20 & 11.2 & 9.1 & 9.3 & 8.9 \\
\hline & & 0.25 & 9.4 & 7.0 & 7.2 & 8.6 \\
\hline & & Total & 11.4 & 9.8 & 8.3 & 11.5 \\
\hline & Total & & 11.1 & 7.6 & 13.0 & 10.8 \\
\hline \multirow[t]{11}{*}{$200-6$} & \multirow[t]{5}{*}{ Low } & 0.1 & 32.2 & 16.6 & 8.5 & 4.9 \\
\hline & & 0.15 & 26.6 & 10.9 & 5.6 & 2.9 \\
\hline & & 0.2 & 26.3 & 9.2 & 3.3 & 1.5 \\
\hline & & 0.25 & 24.9 & 10.3 & 6.1 & -0.7 \\
\hline & & Total & 27.5 & 11.7 & 5.9 & 2.1 \\
\hline & \multirow[t]{5}{*}{ High } & 0.1 & 25.6 & 12.8 & 7.3 & 11.8 \\
\hline & & 0.15 & 24.5 & 8.7 & 8.0 & 6.6 \\
\hline & & 0.20 & 24.9 & 9.5 & 7.0 & 4.6 \\
\hline & & 0.25 & 20.2 & 11.1 & 7.7 & 2.8 \\
\hline & & Total & 23.8 & 10.5 & 7.5 & 6.5 \\
\hline & Total & & 25.6 & 11.7 & 6.7 & 4.3 \\
\hline
\end{tabular}

Table 13

Confidence intervals for the mean $R$.

\begin{tabular}{|c|c|c|c|c|c|}
\hline \multirow[t]{3}{*}{ Distribution } & \multirow{3}{*}{$\begin{array}{l}\text { Flexibility } \\
\text { measure }\end{array}$} & \multicolumn{4}{|c|}{$95 \% \mathrm{CI}$ on mean $R$} \\
\hline & & \multicolumn{2}{|c|}{$n=150, m=5$} & \multicolumn{2}{|c|}{$n=200, m=6$} \\
\hline & & $\begin{array}{l}\text { Lower } \\
\text { bound }\end{array}$ & $\begin{array}{l}\text { Upper } \\
\text { bound }\end{array}$ & $\begin{array}{l}\text { Lower } \\
\text { bound }\end{array}$ & $\begin{array}{l}\text { Upper } \\
\text { bound }\end{array}$ \\
\hline $\begin{array}{l}\text { Norm- } \\
\text { Norm }\end{array}$ & $\frac{w^{2}}{p \Delta L B}$ & 7.6 & 14.6 & 20.3 & 31.0 \\
\hline Tri-Norm & $\frac{w^{2}}{p \Delta L B}$ & 3.1 & 12.1 & 8.0 & 14.2 \\
\hline Exp-Exp & $\frac{1}{p f^{\prime \prime} L B}$ & 6.9 & 19.0 & 1.5 & 11.9 \\
\hline Exp-Norm & $\frac{1}{f^{\prime \prime} L B}$ & 8.6 & 13.0 & 1.0 & 7.6 \\
\hline
\end{tabular}

achieve a significant improvement in the rescheduling cost compared to the SPT rule. From Table 11, we can see how many times each flexibility measure achieves better rescheduling cost than the SPT after rescheduling. $w^{2} / p \Delta L B$ outperformed the SPT rule in 55 cases out of $80 . w / p f^{\prime \prime} L B$ outperforms SPT 56 times.

We next tested proposed algorithm on larger problem size instances with 150 jobs and five machines and with 200 jobs and six machines. For these problems, we only considered the flexibility measures which gave the best mean $R$ value in 100 jobs 3 machines case for each failure-repair time distribution pair. When there are more machines, schedules are generally less vulnerable to a single machine breakdown. This is due to the fact that one can distribute disrupted jobs to more machines which would result in less compression cost. Therefore, different than 100 jobs 3 machines case, we considered MTTR levels of 0.15 and 0.2 in our randomly generated runs. Furthermore, when finding the available capacities of each machine, $D[i]$ ' $\mathrm{s}$, we have used a relatively smaller multiplier 0.1 in the MJA formulation, which was 0.2 in 100 jobs 3 machines case. We again took 10 replications for each setting. Table 12 gives mean $R$ values for selected flexibility measures. Our computational results indicate that the proposed scheduling approach outperforms the SPT rule at different levels of MTTR and match-up time for larger problem instances as well.

Finally, we present the 95\% confidence intervals for mean $R$ values in Table 13 . All of the flexibility measures used in different distribution pairs give a positive lower bound on mean $R$ value, which clearly indicates that our algorithm is significantly better than the SPT rule with respect to minimizing the rescheduling cost.

Using given probability distributions of failure and repair times, we anticipate when and how long each machine could be down and by using designed flexibility measures we schedule the most flexible jobs to the most critical time zones on each machine. Our computational results indicate that combining the proposed probabilistic sequencing idea with proposed flexibility measures are quite efficient in preparing flexible schedules for solving rescheduling cost problems under match-up time limitations. We have tested proposed approach against the SPT sequencing rule and observed a statistically significant difference in rescheduling cost performance. We have also observed that in most of the cases our anticipative scheduling approach performs better than the SPT rule based initial schedules in terms of rescheduling costs. Our results indicate that when the failure-repair behavior pattern is known for a machine, it is quite critical to use the cost function and compression related information in forming initial schedules so that in case of a failure a schedule can be repaired at a reasonable cost. For example, our algorithm outperforms the SPT rule for normal distribution case since proposed downtime probability, $P^{d}(t)$, calculations more accurately capture the disruptive events due to gradual wear (e.g. expected values have an approximately symmetric behavior around a mean value), as opposed to random failures that are represented by an exponential distribution. In the next section, we give concluding remarks.

\section{Conclusion}

In this paper, we have proposed an anticipative scheduling approach for scheduling with controllable processing times. We showed that anticipative decision making in preparing initial schedules can avoid excessive rescheduling costs that may result by reactive processing time adjustments.

We have considered a rescheduling problem to minimize the increase in total manufacturing cost subject to a match-up time constraint. We have designed an anticipative scheduling algorithm which uses proposed flexibility measures that can estimate which jobs can absorb a possible disruption at lowest cost. Proposed algorithm also uses downtime probability functions in determining the job sequence on each machine. Computational results show that considering flexibility measures of jobs and probabilistic nature of machine breakdowns in preparing an initial schedule can significantly improve rescheduling cost performance. As a future research direction, it is possible to consider different reactive scheduling problems in different scheduling environments. This would require developing problem specific flexibility measures. We think that it may also be interesting to consider risky jobs as well as risky machines in preparing initial schedules.

\section{Appendix A. Derivation of $P^{d}(t)$ for the distributions used in the computational study}

Norm-Norm case: In this combination, both failure and repair times are assumed to have a normal distribution. If the failure 
time is expected to be symmetrically distributed around a mean, this combination is suitable. This is actually a realistic case if the machine breakdown is due to a gradual wear process.

Lemma A.1. Let $\mathcal{X} \sim \operatorname{Normal}\left(\mu_{1}, \sigma_{1}\right)$ and $\mathcal{Y} \sim \operatorname{Normal}\left(\mu_{2}, \sigma_{2}\right)$.

$P^{d}(t)=\int_{0}^{\infty} \int_{t-y}^{t} f_{\mathcal{X}}(x) \cdot f_{\mathcal{Y}}(y) d x d y$

where $f_{\mathcal{Y}}(y)=\frac{1}{\sigma \sqrt{2 \pi}} e^{-(y-\mu)^{2} / 2 \sigma^{2}}$

and

$f_{\mathcal{X}}(x)=\frac{1}{\sigma \sqrt{2 \pi}} e^{-(x-\mu)^{2} / 2 \sigma^{2}}$.

Exp-Exp case: Exponential failure time and exponential repair time (Exp-Exp) is widely used in the stochastic literature. Therefore, we considered this case although we do not consider exponential repair time as realistic in our problem. Below, we derive the $P^{d}(t)$ for the Exp-Exp combination.

Lemma A.2. Let $\mathcal{X} \sim \operatorname{Exponential}\left(\lambda_{x}\right)$ and $\mathcal{Y} \sim \operatorname{Exponential}\left(\lambda_{y}\right)$. Then, $P^{d}(t)=\lambda_{x} / \lambda_{y}-\lambda_{x}\left(e^{-\lambda_{x} t}-e^{-\lambda_{y} t}\right)$.

Proof. By Lemma 3.1,

$$
\begin{gathered}
P^{d}(t)=\int_{-\infty}^{t}\left(1-F_{\mathcal{Y}}(t)\right) \cdot f_{\mathcal{X}}(x) d x=\int_{0}^{t} e^{-\lambda_{y}(t-x)} \cdot \lambda_{x} e^{-\lambda_{x} t} d x=\lambda_{x} e^{-\lambda_{y} t} \\
\cdot \int_{0}^{t} e^{\left(\lambda_{y}-\lambda_{x}\right) x} d x=\frac{\lambda_{x}}{\lambda_{y}-\lambda_{x}}\left(e^{-\lambda_{x} t}-e^{-\lambda_{y} t}\right) .
\end{gathered}
$$

Exp-Norm case: Exponential failure is generally a logical approach as it has memoryless property. On the other hand, it may not be appropriate to use exponential repair time since memoryless property may not be suitable in a machining environment. We generally expect to have an approximately symmetric behavior around a mean value when we consider the repair time of a machine. $P^{d}(t)$ of Exp-Norm case can be calculated as below:

Lemma A.3. Let $\mathcal{X} \sim \operatorname{Exponential}(\lambda)$ and $\mathcal{Y} \sim \operatorname{Normal}(\mu, \sigma)$. Then, the down probability is calculated as

$P^{d}(t)=\int_{0}^{t}\left(e^{-\lambda(t-y)}-e^{-\lambda t}\right) f_{\mathcal{Y}}(y) d y+\int_{t}^{\infty}\left(1-e^{-\lambda t}\right) f_{\mathcal{Y}}(y) d y$,

where $f_{\mathcal{Y}}(y)=(1 / \sigma \sqrt{2 \pi}) e^{-(y-\mu)^{2} / 2 \sigma^{2}}$.

Tri-Norm case: This combination is triangular failure time and normal repair time. Tri-Norm is suitable if there is no distribution information for the failures but only the mean values are available.

Lemma A.4. Let $\mathcal{X} \sim$ Triangular $(a, b, c)$ and $\mathcal{Y} \sim \operatorname{Normal}(\mu, \sigma)$. Then,

$$
P^{d}(t)= \begin{cases}\frac{2}{(b-a)(c-a)} \int_{0}^{t-a}\left(\frac{y(2 t-y)}{2}-a y\right) f_{\mathcal{Y}}(y) d y & \\ \quad+\int_{t-a}^{\infty} \frac{(t-a)^{2}}{(b-a)(c-a)} f_{\mathcal{Y}}(y) d y & \text { if } a \leq t \leq c, \\ \int_{t-a}^{\infty} A(t) f_{\mathcal{Y}}(y) d y+\int_{0}^{t-c} B(t, y) f_{\mathcal{Y}}(y) d y & \\ \quad+\int_{t-c}^{t-a} C(t, y) f_{\mathcal{Y}}(y) d y & \text { if } c \leq t \leq b,\end{cases}
$$

where

$$
\begin{aligned}
& A(t)=\frac{\frac{c-a}{b-a}+2\left(b t-t^{2} / 2-b c+c^{2} / 2\right)}{(b-a)(b-c)}, \\
& B(t, y)=\frac{2\left(b y-t y+y^{2} / 2\right)}{(b-a)(b-c)}, \\
& C(t, y)=\frac{2\left(c^{2} / 2-a c-(t-y)^{2} / 2+c(t-y)\right)}{(b-a)(c-a)} \\
& +\frac{2\left(b t-t^{2} / 2-b c+c^{2} / 2\right)}{(b-a)(b-c)} \text {. }
\end{aligned}
$$

From Lemmas A.1-A.4, we see that a closed form expression for $P^{d}(t)$ is only available for the Exp-Exp combination, that might explain why it is widely used in the literature. For the other combinations, $P^{d}(t)$ can only be approximately calculated.

\section{References}

[1] Aytug H, Lawley MA, McKay K, Mohan S, Uzsoy R. Executing production schedules in the face of uncertainties: a review and some future directions. European Journal of Operational Research 2005;161:86-110.

[2] Jensen MT. Improving robustness and flexibility of tardiness and total flowtime job shops using robustness measures. Applied Soft Computing 2001;1:35-52.

[3] Leon J, Wu SD, Storer RH. Robustness measures and robust scheduling for job shops. IIE Transactions 1994;26:32-43.

[4] Mehta SV, Uzsoy RM. Predictable scheduling of a job shop subject to breakdowns. IEEE Transactions on Robotics and Automation 1998;14:365-78.

[5] Leus R, Herroelen W. Scheduling for stability in single-machine production systems. Journal of Scheduling 2007;10:223-35.

[6] Yang B, Geunes J. Predictive-reactive scheduling on a single resource with uncertain future jobs. European Journal of Operational Research 2008; 189:1267-83.

[7] Herroelen W, Leus R. On the merits and pitfalls of critical chain scheduling Journal of Operations Management 2001;19:559-77.

[8] Gürel S, Aktürk MS. Optimal allocation and processing time decisions on nonidentical parallel CNC machines: $\varepsilon$ - constraint approach. European Journal of Operational Research 2007;183:591-607.

[9] Shabtay D, Steiner G. A survey of scheduling with controllable processing times. Discrete Applied Mathematics 2007;155(13):1643-66.

[10] Bean JC, Birge JR, Mittenthal J, Noon CE. Match-up scheduling with multiple resources, release dates and disruptions. Operations Research 1991;39(3):470-83.

[11] Aktürk MS, Görgülü E. Match-up scheduling under a machine breakdown. European Journal of Operational Research 1999;112:81-97.

[12] Aktürk MS, Atamtürk A, Gürel S. Parallel machine match-up scheduling with manufacturing cost considerations. Journal of Scheduling, 2009, to appear, doi:10.1007/s10951-009-0111-2.

[13] Kayan RK, Aktürk MS. A new bounding mechanism for the CNC machine scheduling problems with controllable processing times. European Journal of Operational Research 2005;167:624-43.

[14] Aktürk MS, Atamtürk A, Gürel S. A strong conic quadratic reformulation for machine-job assignment with controllable processing times. Operations Research Letters 2009;37:187-91.

[15] Adiri I, Bruno J, Frostig E, Rinnooy Kan AHG. Single machine flow-time scheduling with a single breakdown. Acta Informatica 1989;26:679-96.

[16] Lee CY, Liman SD. Single machine flow-time scheduling with scheduled maintenance. Acta Informatica 1992;29:375-82. 